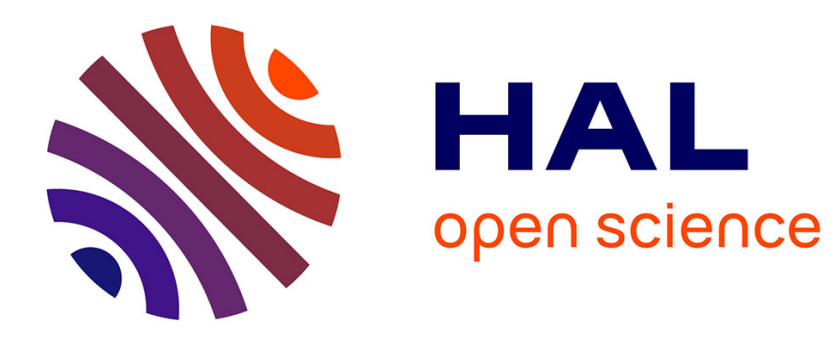

\title{
De la notion de genre appliquée au monde animal
}

Michel Kreutzer

\section{To cite this version:}

Michel Kreutzer. De la notion de genre appliquée au monde animal. Revue du MAUSS, 2012, Que donnent les femmes?, 1 (39), pp.218-235. 10.3917/rdm.039.0218 . hal-01478488

\section{HAL Id: hal-01478488 \\ https://hal.parisnanterre.fr/hal-01478488}

Submitted on 4 Dec 2017

HAL is a multi-disciplinary open access archive for the deposit and dissemination of scientific research documents, whether they are published or not. The documents may come from teaching and research institutions in France or abroad, or from public or private research centers.
L'archive ouverte pluridisciplinaire HAL, est destinée au dépôt et à la diffusion de documents scientifiques de niveau recherche, publiés ou non, émanant des établissements d'enseignement et de recherche français ou étrangers, des laboratoires publics ou privés. 


\title{
De la notion de genre appliquée au monde animal
}

\author{
Michel Kreutzer
}

L'utilisation du terme " genre » relève aujourd'hui si exclusivement du vocabulaire des sciences humaines et sociales qu'il peut paraître insolite qu'un éthologiste suggère d'y recourir pour décrire et analyser la vie de relation des animaux. Telle est toutefois mon intention. Et afin d'étayer cette proposition, je vais tenter de répondre à deux questions qu'elle manque rarement de susciter. La première réclame une définition : qu'est-ce que le genre chez l'animal ? La deuxième interroge le champ d'application : le genre est-il une notion nécessaire, ou, en d'autres termes, celle de sexe ne suffirait-elle pas?

Je vais remettre à plus tard, dans le courant de cet article, la question de la définition, en partie par commodité, mais surtout parce que les sciences nous apportent quotidiennement la preuve qu'il n'est pas nécessaire de définir a priori et avec précision une notion pour l'utiliser efficacement. Prenons l'exemple de la notion de temps (au sens temporel) : quoique le dictionnaire caractérise le temps comme un... " milieu indéfini ", cette absence de définition ne nous empêche nullement de le mesurer et de travailler avec cette notion. Un autre exemple nous est fourni par la notion de conscience, que la philosophie, la psychologie et les neurosciences interrogent incessamment, alors même que nul ne sait clairement ni la définir ni en expliciter les processus.

Quant à la vie sociale des animaux, elle n'échappe pas non plus à ces considérations. Les relations entre individus étaient autrefois décrites en termes d'attraction et de répulsion (tropismes, taxies...), sans pour autant que soient définis le sens précis et la matérialité de ces forces. De fil en aiguille, la valeur heuristique des concepts utilisés a cependant permis d'expérimenter, de tisser des théories, et, aujourd'hui, les théories de l'auto-organisation et de l'intelligence artificielle distribuée sont dérivées de ces mêmes notions d'attraction et de répulsion.

Quant à la seconde question évoquée plus haut, c'est-à-dire : pourquoi en appeler au genre chez l'animal, la notion de sexe ne suffirait-elle pas ? Elle est précisément à l'origine de cet article, et je vais donc m'en expliquer dès à présent.

Depuis quelques décennies, la plupart des auteurs et des chercheurs posent comme une évidence que l'étude du comportement animal relève plus des " sciences de la vie » que des " sciences sociales", et encore moins des "sciences humaines". Remarquons toutefois que ce point de vue n'était sans doute pas celui de la plupart des " savants " du XVIIle siècle et des philosophes des Lumières. Ces derniers, s'interrogeant sur " l'ordre de la nature ", n'hésitaient pas à mettre en proximité leurs conceptions de l'animal et de l'humain et c'est cette démarche qui a permis l'élaboration de la théorie de l'évolution, applicable à l'homme et l'animal, ainsi qu'aux notions darwiniennes de sélections naturelle et sexuelle.

On sait aussi que, depuis le XIXe siècle, une tradition académique de recherche en psychologie analyse les continuités et discontinuités entre l'animal et l'humain : la psychologie comparée. En outre, les recherches récentes de primatologues sur les « cultures » animales empruntent beaucoup à l'ethno-anthropologie, quand elles ne sont pas directement conduites par des chercheurs de ces 
disciplines. Et le recours aux sciences sociales et économiques est manifeste dans la théorie éthologique dite behavioural ecology [Krebs \& Davies, 1978], qui décrit les animaux comme des agents gérant des budgets " temps-énergie " afin d'atteindre des objectifs grâce à des stratégies qui minimisent les coûts et optimisent les bénéfices. Pas plus aujourd'hui qu'hier, la conception de la vie animale n'est en aucune façon la propriété exclusive d'un point de vue "naturaliste ", et de nombreux concepts issus des sciences sociales sont régulièrement sollicités pour enrichir et affiner cet entendement. Notons qu'à l'inverse, un point de vue éthologique et évolutionniste peut être appelé pour décrire et analyser les appariements chez l'humain [Kreutzer, Augustins, 2012].

À quoi s'intéressent les éthologistes quand ils décrivent la vie animale ? Ils nous montrent des individus qui mangent, dorment, se querellent et se battent, paradent, séduisent, copulent, élèvent et protègent des jeunes, sont malades et vieillissent. Bref, des individus qui vivent, s'organisent, se conservent, se reproduisent et meurent. À côté de ces descriptions " concrètes ", les éthologistes recourent à des conceptualisations pour décrire la vie et la socialité animale : nous disons que les individus communiquent, gèrent des situations de conflit et de coopération, possèdent des systèmes d'appariement monogames ou polygames, vivent parfois en groupes sociaux hiérarchisés, se constituent des territoires, s'entraident et font preuve d'altruisme envers des apparentés et des familiers dans le cadre de systèmes d'alliances. Nous voyons bien que le vocabulaire « humanise le bestiaire " décrit et analysé, quand bien même l'approche se donne pour " biologique ». Les études éthologiques consacrées aux activités sexuelles et de dominance des animaux sont du reste si nombreuses qu'on pourrait se demander si la pertinence des questions traitées réside dans la réalité de la vie animale ou bien dans le regard de l'observateur. Afin d'analyser au plus près l'intérêt et les limites d'études sur le " sexe et le pouvoir ", nous allons commencer par en évaluer la pertinence.

\section{Le sexe}

Fondamentalement, en biologie animale, le sexe désigne un mode de reproduction opposé à la reproduction asexuée. En reproduction sexuée, il est nécessaire de fabriquer des gamètes, ovules et spermatozoïdes; ensuite, ces gamètes doivent se rencontrer pour former une cellule-œuf, qui permettra le développement d'un nouvel organisme. Des organismes fabriquent et portent ces gamètes et les mettent en contact. On nomme mâles ceux qui portent les spermatozoïdes, et femelles celles qui portent les ovules. Cependant, à peine a-t-on posé ce distinguo essentiel que les choses se compliquent : non seulement certains individus peuvent, à un certain moment, être porteurs d'ovules, et à un autre, de spermatozoïdes (on dit alors qu'ils changent de sexe), mais encore d'autres portent à la fois des spermatozoïdes et des ovules (on dit alors qu'ils sont hermaphrodites).

Par ailleurs, le terme de sexe est utilisé dans des domaines aussi divers que l'anatomie, la physiologie, les activités sociales, parentales, reproductrices, les orientations sexuelles... Mais alors, quoique cette notion se décline le plus souvent en termes de mâle(s) et de femelle(s) appliqués à des individus qui vont mettre en contact leurs gamètes, le terme " sexe " désigne des individus et des comportements, c'est-à-dire bien autre chose que ce qui est à l'œuvre dans les combinaisons de gamètes de la reproduction sexuée [Giraldeau, 2009].

En éthologie, voire en psychologie animale et humaine, on a souvent recours aux termes " mâle » et " femelle » pour définir des types de comportement et des catégories mentales qui sont liés à la reproduction et aux comportements parentaux. Le vivant est alors conçu comme animé par un programme sexué répondant à un ordre de la nature qui tend à reproduire et à transmettre, via des mâles et des femelles. Les concepts issus des sciences sociales qu'utilisent les éthologistes, et que nous évoquions au début de cet article, se juxtaposent alors aux catégories habituelles des sciences 
de la nature, et se combinent avec elles de telle manière que les descripteurs sociaux semblent relever de l'ordre de la nature. Une grande partie de la vie sociale apparaît comme le résultat des interactions d'agents dont la caractéristique principale est le sexe, réparti en deux catégories : celle des mâles et celle des femelles.

\section{Le pouvoir}

\section{Dominance et possession}

L'étude de la vie sociale des animaux, notamment celle des primates, a très rapidement mis en évidence la prééminence de certains individus sur les autres. Cette dominance apparaît souvent comme le résultat de conduites agressives, de manifestations d'intimidation. Les observateurs ont ainsi été conduits à décrire les « sociétés animales " comme des organisations hiérarchiques dans lesquelles, grâce à un rang élevé, des individus contrôlent les activités des autres et se procurent des avantages, notamment pour se nourrir et se reproduire. On parle aussi de "leadership » lorsqu'un individu, mâle ou femelle, est suivi par les autres ou fait preuve d'autorité lors des déplacements en groupe ou lors de la recherche de nourriture.

Mais dans la vie des animaux comme dans la nôtre, le pouvoir ne consiste pas seulement à dominer les autres ; il se décline aussi en termes de possession. Alors que la domination s'exerce sur des individus, la possession porte sur des biens, des objets, de l'espace. C'est ainsi que chez des espèces qui ne vivent pas en grands groupes sociaux mais en couple, ce qui est le cas de nombreuses espèces d'oiseaux, des individus s'emparent d'espaces, appelés territoires, dont ils excluent leurs congénères. Ces espaces, souvent établis et défendus par les mâles, constituent aussi des réserves de nourriture, des lieux de reproduction et d'élevage des jeunes. Dans cette perspective, le pouvoir peut être considéré comme une fonction relevant des propriétés fondamentales du vivant, c'est-à-dire : se conserver, s'organiser et se reproduire. De ce point de vue, la vie sociale prend la forme de conflits d'intérêt entre les individus.

\section{Coopération}

Cependant le conflit d'intérêts n'est pas le seul déterminant de la vie sociale animale. Les éthologues ont toujours décrit des phénomènes de coopération entre individus. Non seulement entre mâles et femelles, au sein des couples, pour se reproduire, élever les jeunes et conserver des espaces, mais encore au sein de grands groupes sociaux. Dans certaines organisations de primates, on peut observer des comportements d'apaisement et de réconciliation qui permettent à des individus de renouer des liens après un conflit [Thierry et al., 2008]. La vie en groupe ne peut se permettre des conflits permanents entre individus. Se déplacer, se préserver des prédateurs, éviter les groupes de congénères rivaux nécessite une cohésion du groupe, ce qui implique la réduction des antagonismes et la coopération. Du reste, pour mieux asseoir leur pouvoir, les individus peuvent aussi choisir de contracter des alliances et de s'assurer des coopérations. L'union fait la force, et elle permet de mieux gérer les conflits d'intérêt. La communication entre les individus assure alors la régulation des coopérations et des conflits.

\section{Altruisme}

Les comportements dits " altruistes ", où un individu va en partie sacrifier ses chances de survie et de reproduction au profit d'un autre, constituent un remarquable exemple de coopération et d'entraide. On sait que Darwin s'interrogeait sur les faits qui pouvaient prendre en défaut sa théorie de la sélection naturelle et sexuelle. L'existence de stériles chez les insectes sociaux était l'un de ces phénomènes. En effet, si ce qui est " gagnant ", au cours de l'évolution, c'est de vivre longtemps en bonne santé et d'être un bon reproducteur, l'existence chez les insectes sociaux de tant d'individus 
stériles qui s'occupent du couvain paraît pour le moins paradoxale. Il a fallu attendre Hamilton [1964a, 1964b] pour que la théorie de la sélection naturelle et sexuelle soit complétée par celle de « sélection de parentèle ", qui soutient que l'altruisme n'entrerait pas en contradiction avec la théorie darwinienne dès lors que la diminution des chances de survie et de reproduction de certains individus augmente celles d'individus apparentés.

Dans une telle perspective, les comportements altruistes ne devraient cependant exister qu'à l'égard des apparentés. Or si nombre d'expérimentations et d'observations corroborent cette conception, il est des cas où des individus diminuent leurs chances de survie et de reproduction au profit de nonapparentés. Mais point n'est besoin en l'occurrence de déroger aux principes de la sélection de parentèle : une fois que l'altruisme entre apparentés est apparu, rien n'empêche que cette propriété ait été utilisée dans un autre cadre que celui où elle a été sélectionnée à l'origine et pour d'autres raisons. De la même manière que chez l'humain, la main peut être utilisée pour écrire et jouer du piano, alors qu'il ne s'agit pas là des raisons qui ont prévalu lors de sa sélection chez nos ancêtres primates et hominidés.

Quant à savoir si des individus en aident d'autres en échange d'une aide en retour, de nombreux travaux portent sur ce thème dit de l'altruisme réciproque. Mais Alain Caillé [2009] souligne qu'il pourrait s'agir là d'un équivalent du " don », un phénomène qui, selon Mauss [1924], est, avec l'« échange ", au cœur de la vie sociale des humains. Nous abordons là la question de la gratuité du don, un débat qui anime depuis longtemps l'univers des éthologistes et des sociologues. Chez l'humain, si certains auteurs voient dans cette dynamique un " je te donne aujourd'hui pour recevoir demain ", on ne peut toutefois pas écarter un renforcement narcissique du donneur, son geste augmentant l'estime qu'il se porte à lui-même. Mais dans le cas des animaux, on devra, faute de pouvoir questionner leur subjectivité, se contenter d'envisager que l'entraide et l'altruisme se maintiennent parce qu'ils renforcent la vie du groupe et sa cohésion, en établissant et en consolidant des liens qui contrebalancent la compétition interindividuelle. Des éthologistes étudient aujourd'hui la sociabilité des animaux, et particulièrement celle des primates, afin de vérifier si des échanges de biens (nourriture) et de services (épouillage) sont réalisés avec un souci de réciprocité [Dufour et al., 2009]. Une réponse affirmative nous conduirait à postuler l'existence d'un Animus œconomicus ayant une certaine forme de rationalité. Si ces hypothèses devaient se confirmer, les auteur(e) s ne manqueraient pas, lors de prochains travaux, de les appliquer aux aspects cognitifs des relations entre les femelles et les mâles.

Au total, la vie sociale est agencée de telle sorte que, bien souvent, les avantages des uns constituent des désavantages pour les autres. L'intérêt qu'ont les individus à se conserver et à se reproduire conduit de fait à une vie de relation qui est l'objet d'incessantes interactions conflictuelles. Cette vie, que caractérise un équilibre instable, où alternent construction et déconstruction, n'apparaît pas de tout repos. L'ambivalence des attitudes en constitue le cœur.

\section{Le genre}

Chez l'humain, les normes et identités enregistrent, fixent, transmettent, proposent, imposent des représentations du genre, et les recherches sur le genre se sont depuis de nombreuses années émancipées de celles portant sur le sexe. Le sexe et le pouvoir se manifestent par les efforts dont font preuve les individus pour s'exhiber et parader, mais aussi pour s'attribuer les objets, espaces et statuts qui les rendront désirables aux yeux des congénères. Ces activités leur permettent de consommer des jouissances qui, bien entendu, assurent la conservation et la reproduction, mais aussi de construire des rôles sociaux, parmi lesquels les propriétés sociales du genre se constituent sur la base de l'opposition masculine-féminine. 
Les propriétés sociales étudiées grâce à la notion de genre existent bien dans le contexte de la vie sociale d'espèces animales. Cependant, si, chez l'animal, le sexe fait l'objet de nombreuses études, aussi bien en ce qui concerne la diversité des mécanismes qui le déterminent qu'en ce qui concerne les fonctions reproductrices des porteurs d'ovules et de spermatozoïdes, le genre est une notion très généralement écartée. Pourtant, dès lors qu'on aborde l'étude de la diversité des fonctions sociales, des activités coopératives et conflictuelles entre " mâles » et " femelles ", les comportements parentaux, le genre semble prendre tout son sens. Un certain nombre d'activités sociales des animaux ne sauraient être décrites en faisant référence aux seules notions de mâle ou de femelle. Je vais ci-dessous en donner des exemples.

\section{Comportements parentaux}

Monogamiques ou polygamiques (polygynes ou polyandres), les structures reproductrices permettent une grande variété de comportements parentaux. Chez un très grand nombre d'espèces, il n'existe pas de comportement maternel ou paternel prédéfini. Par exemple, chez les poissons d'eau douce, on rencontre le plus fréquemment la monogamie avec des soins biparentaux. Mais chez les poissons marins, dans les cas où on peut observer des soins parentaux, ils sont le fait des mâles. Chez les oiseaux, $80 \%$ des espèces sont monogames, avec beaucoup de soins biparentaux. Chez les mammifères, dans $80 \%$ des cas, les structures sont polygames. Les femelles allaitent, et $95 \%$ d'entre elles s'occupent des petits. Mais des mâles peuvent participer : chez les carnivores et les primates, on relève des soins paternels dans $20 \%$ des cas, avec de grandes différences individuelles. La répartition des rôles parentaux subit des variations notables liées aux stratégies reproductrices individuelles. La connaissance du sexe des individus ne permet donc pas de leur attribuer ipso facto un rôle parental typique.

Les rôles sociaux ne sont pas uniquement définis par des relations de pouvoir ; les rôles parentaux chez les mammifères en apportent à mon sens une preuve explicite. Les mâles ne pouvant allaiter, leurs relations avec les nouveau-nés sont de ce fait bien différentes de celles des femelles. Mais chez les oiseaux, où mâles et femelles peuvent construire les nids, couver les œufs et nourrir les jeunes, les rôles parentaux peuvent se distribuer différemment de ceux des mammifères.

Chez les oiseaux de l'espèce " paruline masquée ", tandis que les mâles les plus attractifs consacrent beaucoup de temps à la recherche de nombreuses femelles partenaires, les autres mâles s'investissent dans les soins parentaux avec une seule femelle. On parle alors de trade-off, c'est-àdire d'un compromis entre l'investissement parental et la désirabilité des mâles par les femelles. Chez un autre oiseau chanteur, le rouge-queue, mâles et femelles s'occupent des petits lorsqu'ils sont au nid. Mais après l'envol, une partition de la nichée s'opère chez $20 \%$ des couvées. Une partie des jeunes suivent le mâle, sont uniquement nourris par lui et ne répondent plus qu'à ses appels, alors que les autres suivent la femelle. Ici encore, le sexe des individus ne permet pas de définir le rôle parental que vont exercer les individus.

\section{Orientation sexuelle}

Dans leurs descriptions de l'homosexualité animale, les auteur(e) s ont bien souvent privilégié la monte entre mâles [Sommer \& Vasey, 2006]. Mais Bagemihl [2000] rapporte quantité d'observations menées depuis très longtemps chez un très grand nombre d'espèces, où on relève une grande variété de comportements orientés vers des congénères du même sexe. À savoir des parades de cour, des contacts physiques à connotation sexuelle habituellement décrits lors de copulations entre partenaires hétérosexuels, des attachements durables et même des comportements parentaux.

Dans cet impressionnant inventaire, je relèverai des exemples chez les oiseaux : pour être moins connus, ces phénomènes n'y sont pas moins spectaculaires. Chez les laridés (goëland), des femelles 
forment des couples de "lesbiennes » [Bagemihl, 2000, p. 545- 547 ; Conover, 1989] ; lorsque l'une des femelles (quand ce ne sont pas les deux) s'en va copuler avec un mâle, aucun lien durable ne s'installe pour autant avec lui : une fois leurs ovules fécondés, elles nidifient ensemble et élèvent leurs jeunes sans aide extérieure. Chez les cygnes noirs, les couples de mâles " gay " font de même [Bagemihl, 2000, p. 488-491; Ritchie, 1926] : ils s'accaparent des espaces favorables, et, après s'être temporairement associés avec une femelle et l'avoir fertilisée, ils la chassent une fois qu'elle a pondu ses œufs pour élever les jeunes seuls et avec succès.

L'orientation homosexuelle fait l'objet de nombreuses études et elle suscite quantité d'hypothèses qui opposent souvent un déterminisme génétique et physiologique [Balthazart, 2010] à une origine acquise au cours du développement psychologique et comportemental [voir sur ce point Poiani, 2010]. En recherchant des bases anatomiques et physiologiques à cette orientation, on a pu remarquer que, chez les rats et furets mâles à orientation hétérosexuelle, l'aire préoptique médiane présente un volume supérieur à ce qu'on observe d'une part chez les mâles à orientation homosexuelle, et de l'autre chez les femelles. L'inverse est constaté pour les femelles. Dans le cas du mouton, où on a noté une orientation homosexuelle chez environ $8 \%$ des mâles, ces béliers possèdent une aire préoptique plus petite que celle qui est habituellement constatée chez les mâles à orientation hétérosexuelle.

\section{La dominance, la hiérarchie et les conflits d'intérêt}

Comme je l'ai mentionné plus haut, la coopération et les conflits animent la vie de relation des animaux. Les alliances sont des stratégies qui permettent à des groupes d'individus de manipuler des congénères afin d'en obtenir des avantages. Ces processus existent dans les groupes animaux, aussi bien chez les mâles que chez les femelles. La hiérarchie entre les mâles et les femelles a été décrite maintes fois, par exemple chez les primates. Elle donne à des individus la possibilité d'en asservir d'autres.

La question de l'origine de cette autorité, qui peut recourir au " consentement ", assorti ou non de fortes contraintes, commence à être bien documentée; elle a été étudiée notamment dans le contexte où des femelles dominent ou assurent un leadership sur les mâles. Le phénomène est bien connu chez les lémuriens [Jacobs et al., 2008] et chez certaines espèces de gibbons [Barelli et al., 2008], où les femelles sont prioritaires sur les mâles dans la conduite des groupes et l'accès à la nourriture.

Dès le début des années 1970, de nombreuses études se sont consacrées aux relations de dominance entre mâles et femelles chez certaines espèces de babouins [Kummer et al., 1970 ; Nagel, 1973]. En Éthiopie, deux d'entre elles, Papio hamadryas et Papio anubis, présentent des organisations sociales bien différentes. Les hamadryas vivent en grandes tribus regroupant de petites unités (dites unimâles) dont chacune est composée d'un mâle adulte dominant et de femelles avec leurs jeunes. Plutôt tyranniques, ces mâles ne tolèrent pas que les femelles s'éloignent : ils pratiquent une continuelle activité de regroupement, avec menaces et rétorsions physiques à l'endroit des contrevenantes. Les mâles anubis, quant à eux, sont beaucoup plus tolérants ; ils vivent en groupes composés de plusieurs mâles et femelles (dits multimâles-multifemelles), sans comportement de harcèlement ni de regroupement envers elles. Or il n'est pas rare d'observer dans la nature des groupes mixtes, composés d'hamadryas et d'anubis et d'individus-hybrides issus de croisement entre les deux espèces. La structure de ces groupes mixtes offre une grande variété de situations pour les femelles. La liberté qu'elles peuvent avoir d'aller et venir et de copuler avec différents mâles dépend principalement du nombre de mâles qui pratiquent des comportements de regroupements typiques des hamadryas. Le comportement des femelles n'est donc déterminé ni par leur espèce ni par leur 
sexe, mais par la structure sociale des groupes auxquels elles appartiennent et par les comportements de domination des mâles.

Un remarquable exemple de conflit d'intérêts entre mâles et femelles nous est fourni par le gobemouche noir (Ficedula hypoleuca), une espèce d'oiseau chanteur qui vit en couple [Temrin \& Arak, 1989]. Au début du printemps, lors de leur retour de migration hivernale qui se fait en groupes souvent unisexés, les mâles arrivent les premiers sur les sites de reproduction. Ils deviennent alors intolérants les uns avec les autres, et s'installent chacun dans des espaces qu'ils défendent contre l'intrusion de leurs congénères mâles. De l'intérieur de ces territoires, ils leur signalent leur présence par leurs chants, et on a pu montrer qu'un individu s'attaque à un haut-parleur situé sur son territoire dès lors qu'il diffuse des enregistrements de chants de sa propre espèce. Chaque individu est donc bien en compétition avec tous ceux qui recherchent les mêmes biens que lui, c'est-à-dire tous ceux qui ont les mêmes habitudes de vie. En l'occurrence, un individu de la même espèce constitue le concurrent le plus redoutable.

Puis, les territoires étant constitués, les femelles reviennent à leur tour de migration, environ une semaine après les mâles. En les entendant chanter, elles vont s'employer à rechercher un partenaire. Elles visitent les territoires, écoutent les chants, observent les parades, et choisissent avec lequel s'apparier. Au cours de cette phase, ce sont les femelles entre elles qui se trouvent en compétition, car certains mâles sont plus convoités que d'autres. Mais mon propos n'est pas de détailler ici ces phases d'appariement, par ailleurs bien étudiées; je cherche à mettre l'accent sur un autre processus. Certains mâles (jusqu'à $15 \%$ ou plus dans une même population) s'installent dans deux territoires éloignés de quelques centaines de mètres, vaquant de l'un à l'autre pour y chanter et en défendre l'accès aux congénères mâles. Ils vont tenter $d$ 'attirer des femelles différentes dans chacun de ces territoires, afin de s'y reproduire et de manière à posséder deux nichées, au lieu d'une seule comme la plupart des autres mâles. Du point de vue de la reproduction, on constate que leur succès est supérieur à celui des mâles monoterritoriaux et monogames. Mais du point de vue des femelles, le succès reproducteur de celles qui sont appariées à des mâles polyterritoriaux et polygames est globalement moindre. Sans doute parce que le mâle qui partage son temps et son activité entre deux territoires consacre moins de temps à chaque couvée (pour nourrir les jeunes, avertir la nichée de la présence d'un prédateur...). Dans ce cas, le conflit d'intérêts entre mâles et femelles est manifeste : les mâles ont intérêt à être polygames, et les femelles à ne pas choisir de tels mâles. Les femelles, au moment des appariements, tireraient donc avantage à déterminer si les mâles sont ou non polyterritoriaux.

\section{Modèles de masculinité}

Les études sur les choix de partenaire(s) s'appuient souvent sur la théorie de la sélection sexuelle. Cette dernière postule que ces choix seraient contraints non seulement par les préférences que les femelles auraient pour certains types de mâles, mais aussi par les compétitions que se livrent les mâles afin de se présenter d'une manière favorable pour choisir ou être choisi. Cependant, comme le prévoyait Darwin, les études des éthologistes montrent que, chez les mammifères et les oiseaux, les femelles sont plus sélectives et attentives au choix de leur(s) partenaire(s) que les mâles.

En effet, dans des structures reproductrices où les femelles dépensent plus de temps et d'énergie que les mâles pour élever les jeunes, elles ont plus à perdre que les mâles au terme d'un échec reproducteur. Dans toute " affaire ", c'est celui qui investit le plus qui a le plus à perdre en cas de faillite. Sous un autre angle, on peut aussi considérer que, lors de la reproduction, les spermatozoïdes étant disponibles en plus grand nombre que les ovules, ces derniers constituent une ressource rare. II en résulte que les femelles disposent de moins de « tickets " reproducteurs que les mâles, et que, à chaque fois qu'elles en utilisent un, elles ne peuvent le gaspiller aussi dispendieusement que les 
mâles sont à même de le faire. Elles doivent donc être attentives à son utilisation afin d'optimiser au mieux son usage.

Les auteurs postulent que, pour réaliser ces choix, les femelles disposent de modèles de mâles désirables, avec lesquels la reproduction est plus efficace. Chez les oiseaux chanteurs, des études ont montré que les qualités du territoire, du physique, des chants et des parades des mâles déterminent le choix des femelles [Anderson, 1994], et que, le plus souvent, ces dernières accordent de manière similaire leur préférence aux mêmes caractéristiques. Il existe ainsi des mâles plus attractifs que d'autres, ceux qui sont à même de déployer des signaux " honnêtes ", c'est-à-dire difficiles à contrefaire par des mâles de moindre qualité. Chez les oiseaux, et plus précisément chez le canari (Serinus canaria), nous avons pu montrer que les femelles accordent toutes une prédilection marquée à une structure particulière des chants de mâles appelée la séquence $\mathrm{A}$ [Vallet \& Kreutzer, 1995]. Cette phrase du chant possède la particularité d'être difficile à chanter, car elle nécessite une étroite coordination des bronches droite et gauche de la syrinx, organe du chant chez les oiseaux [Suthers et al., 2004] ; de plus, les deux hémisphères cérébraux impliqués dans la production du chant doivent également coordonner leurs activités [Hallé et al., 2003], si bien que ces performances motrices peuvent être comparées à un travail de jongleur qui coordonnerait très rapidement et de manière asynchrone ses mains droite et gauche. Des " superstimuli » ont même pu être synthétisés et produire des attractions supérieures à celles de signaux naturels [Draganoiu et al., 2006]. La séquence A s'est également révélée être un bon indice du statut hiérarchique des mâles [Parisot, 2004]. De telles études permettent d'affirmer que, pour les femelles, dans la catégorie des partenaires potentiels, certains individus sont plus désirables et plus prototypiques que d'autres. II s'agit à mon sens de représentations mentales, qui s'apparentent à celle de " masculinité » dans les études sur le genre chez l'humain.

\section{Chez l'animal comme chez l'humain...}

Pour conclure et répondre à notre questionnement liminaire : les animaux ont-ils un genre ? je remarquerai simplement que lorsque des études chez l'humain interrogent la masculinité et/ou la féminité, la distribution des rôles parentaux, l'orientation sexuelle, la hiérarchie entre les hommes et les femmes, on dit qu'elles interrogent le genre. De ce fait, si les mêmes propriétés sociales sont interrogées chez l'animal, il m'apparaît tout à fait loisible de penser que c'est également le genre qu'on interroge chez les animaux.

Maintenant, revenons-en au problème que j'ai écarté au début de ce texte : comment définir le genre chez l'animal et le différencier du sexe?

Notons tout de suite qu'il ne peut y avoir de genre, chez l'animal comme chez l'humain, que s'il y a du sexe. On ne saurait concevoir la notion de genre dans un contexte de reproduction asexuée. $S^{\prime}$ il ne se confond pas avec lui, le genre s'étaie donc sur le sexe. Le genre, comme le sexe, s'appuie sur de l'observable. Mais le genre n'a pas de caractère essentialiste ; c'est une notion qui permet de décrire des rôles sociaux, des rapports de pouvoir, des conflits et coopérations entre mâles et femelles; bref, des propriétés de la vie de relation. Le genre appliqué à l'animal a donc un rôle descriptif, et non pas normatif. En donnant une priorité à l'étude des propriétés plutôt qu'à celle de la catégorie " genre ", nous souscrivons au principe énoncé par Priscille Touraille [2009, p. 473] :

"Seules [des] propriétés constituent ce que l'on peut appeler des "réalités". À chaque fois que des biologistes partent des catégories au lieu de partir des propriétés, cela suscite plus de problèmes dans la description de la réalité que cela n'aide à l'expliquer. » 
Le genre, chez l'animal comme chez l'humain, constitue un champ d'études où l'on interroge à la fois " le sexe social » et les relations de pouvoir qui lui sont liées. Il s'agit à mon sens d'une propriété générale liée à la sociabilité et à ses caractères et fonctions. Je dirai donc que le genre est un champ d'études regroupant l'ensemble des rôles sociaux et des relations de pouvoir où mâles et femelles sont impliqués. Là où des êtres font preuve de sociabilité, remplissent des rôles, exercent du pouvoir, sont en conflit et coopération, il y aurait, y compris chez l'animal, du genre. Je propose donc que la notion de genre ne soit pas spécifique à l'humain. II m'apparaît en outre que l'extension du domaine d'application de cette notion à la vie sociale des animaux en renforce la pertinence.

En avançant cette idée, je ne vise en aucun cas à naturaliser ce concept. Comme je l'ai déjà montré au cours de cet article, ce sont plutôt les sciences sociales qui investissent le champ de l'éthologie. En outre, je souscris pleinement aux raisons qui conduisent certains auteurs à redouter une naturalisation des rôles sociaux chez l'humain. Cette conception a en effet trop souvent conduit à considérer que des différences et des inégalités sociales, notamment entre les hommes et les femmes, sont naturelles et donc "normales ", alors même que la démonstration des supériorités masculines s'est révélée comme le résultat d'expériences erronées [Vidal, 2005].

À l'inverse de ce danger de naturaliser l'humanité, se profile le risque d'humaniser notre bestiaire et de tomber dans une forme d'anthropomorphisme. En l'occurrence, dans la mesure où personne n'échappe au lot commun, mon regard profane et savant n'est-il pas sous l'influence de l'idéologie régnante ? Comme le constate Claude Lévi-Strauss [1952, p. 43-44] :

" Dès notre naissance, l'entourage fait pénétrer en nous, par mille démarches conscientes et inconscientes, un système complexe de références consistant en jugements de valeurs, motivations, centres d'intérêt... "

Mais au-delà de ces considérations dont je ne saurais sous-évaluer les conséquences, j'estime que c'est la valeur opératoire de la notion de genre, sa capacité à donner de l'intelligibilité aux faits, qui en vérifieront la pertinence et en généraliseront l'usage en éthologie. Autrement dit, c'est sur sa valeur heuristique qu'elle sera validée.

L'éthologie renouant une fois encore avec les emprunts qu'elle est accoutumée de faire aux sciences sociales, le mouvement oscillant qui consiste tantôt à humaniser le bestiaire et tantôt à naturaliser I'humain rend instables les frontières entre les sciences de la nature et les sciences humaines et sociales. Il faut s'y faire car, depuis la théorie darwinienne, les limites entre nature et culture n'ont cessé de se déplacer, de se construire et se déconstruire. Force est de constater, aujourd'hui comme hier, qu'elles ne sont peut-être pas où on les voyait, ni telles qu'on les imaginait.

\section{Références bibliographiques}

ANDERSON M., 1994, Sexual selection. Princeton University Press, Princeton.

BAGEMIHL B., 2000, Biological Exuberance, Stonewall Inn Editions.

BALTHAZART J., 2010, Biologie de l'Homosexualité, Éditions Mardaga, Wavre, Belgique.

BARELLI C., BOESCH C., HEISTERMANN M., REICHARD U.H., 2008, « Female white-handed gibbons (Hylobates lar) lead group mouvements and have priority to access to food resources ", Behaviour, $n^{\circ} 145$, p. 968-981. 
CAILLÉ A., 2009, Théorie anti-utilitariste de l'action. Fragments d'une sociologie générale, La Découverte/MAUSS, Paris.

CONOVER M.R., 1989, « Parental care by male-female and female-female pairs of ringed-billed gulls », Colonial waterbirds, $n^{\circ} 12$, p. 148-151.

CORDS M. \& AURELI F., 2000, Reconciliation and Relationship Quality, in Natural Conflict Resolution, p. 177-198, University of California Press, Berkeley.

DARWIN C., 1859, On the Origin of Species by Means of Natural Selection, or the Preservation of Favoured Races in the Struggle for Life, John Murray, London.

DARWIN C., 1871, The Descent of Man and Selection in Relation to Sex, John Murray, London.

DRAGANOIU T., NAGLE L., KREUTZER M., 2002, « Directional female preference for an exaggerated male trait in canary (Serinus canaria) song ", Proceedings of the Royal Society, London, $n^{\circ} 269, p$. 2525-2531.

DRAGANOIU T., NAGLE L., MUSSEAU R., KREUTZER M., 2006, « In a song bird, the black redstart, parents use acoustic cues to discriminate between their different fledgings ", Animal Behaviour, $\mathrm{n}^{\circ}$ 71 , p. 1039-1046.

DUFOUR V., PELÉ M., NEUMANN M., THIERRY, B. \& CALL, J., 2009, « Calculated reciprocity after all : computation behind token transfers in orang-utans ", Biology Letters, $n^{\circ}$ 5, p. 172-175.

GIRALDEAU L.-A., 2009, "Comportements sexuels au sein du règne animal », in GOUYON P.-H. (dir.), Aux origines de la sexualité, chap. XI, Fayard, Paris, p. 172-193.

HALLÉ F., GAHR M., KREUTZER M., 2003, « Effects of unilateral lesions of HVC on song patterns of male domesticated canaries ", Journal of Neurobiology, $n^{\circ} 56$, p. 303-314.

HAMILTON W.D., 1964a, " The genetical evolution of social behaviour, I. », Journal of Theorical Biology, $n^{\circ}$ 7, p. 1-16.

$-1964 b$, "The genetical evolution of social behaviour, II. », Journal of Theorical Biology, $n^{\circ} 7, p$. $17-52$.

JACOBS A., MAUMY M., PETIT O., 2008, « The influence of social organisation on leadership in brown lemers (Eulemur fulvus fulvus) in a controlled environnement ", Behavioural processes, $n^{\circ} 79$, p. 111-113.

KREBS J.D.N., 1978, Behavioural Ecology, An Evolutionary Appraoch, Blackwel scientific Publications, Oxford.

KREUTZER M., AUGUSTINS G., 2012, « Les appariements chez l'animal et l'humain : satisfactions individuelles et comportements sociaux », Ethnologie Française, XLII (42), $n^{\circ} 3$.

KUMMER H., GOETZ W., ANGST, W., 1970, "Cross species modification of social behavior, in baboon ", in NAPIER J.R. \& NAPIER P.H., Old World Monkeys : Evolution, Systematiccs and Behavior, Academic Press, London, p. 351-363.

LÉVI-STRAUSS C., 1952, Race et histoire, Denoël, Paris.

MAUSS M., 1924, «Essai sur le don », L’Année sociologique, n 1, p. 30-186. 
NAGEL U, 1973, «A comparison of anubis baboon, hamadryas baboon and their hybrids at a species border in Ethiopia », Folia primatologica, n 19, p. 104-165.

PARISOT M., NAGLE L., VALLET É., KREUTZER M., 2004, « Dominance-related foraging in female domesticated canaries under laboratory conditions ", Canadian Journal of Zoology, n 82, p. 12461250.

POIANI A., 2010, Animal Homosexuality, a biosocial perspective, Cambridge University Press, Cambridge.

RICHIE J.-P., 1926, « Nesting of two male swans », Scottish Naturalist, n 159, p. 95.

SILK J., ALTMANN J., ALBERTS S.C., 2006, " Social relationship among adult female baboons (Papio cynocephalus), Variation in the strength of social bonds ", Behavioural Ecology \& Evolution, $n^{\circ}$ 61, p. 183-195.

SOMMER V., VASEY P.L., 2006, Homosexual Behaviour in Animals, an evolutionary perspective, Cambridge University Press, Cambridge.

SUTHERS R., VALLET É., TANVEZ A., KREUTZER M., 2004, « Bilateral song production in domestic canaries ", Journal of Neurobiology, vol. 60, $n^{\circ} 3$, p. 381-393.

TEMRIN H., ARAK A., 1989, « Polyterritoriality in passerine birds », Trends in Ecology and Evolution, $\mathrm{n}^{\circ} 4$, p. 106-109.

THIERRY B., 2004, "Social epigenesis ", in THIERRY B., SINGH M., KAUMANNS W., Macaque Societies : A Model for the Study of Social Organisation, Cambridge University Press, Cambridge, p. 267-289.

THIERRY B., AURELI F., NUNN C.L., PETIT O., ABEGG G., DE WAAL F.B.M., 2008, « A comparison study of conflict resolution in macaques, insights into the nature of trait covariation ", Animal Behaviour, $n^{\circ} 75$, p. $847-860$.

TOURAILLE P., 2009, Genre et sexe : sortir de l'imbroglio conceptuel, chap. XXV, p. 466-489, in GOUYON P.-H., Aux origines de la sexualité, édité par Pierre-Henri, Fayard, Paris.

VALLET É., KREUTZER M., 1995, « Female canaries are sexually responsive to special song phrases ", Animal Behaviour, n 49, p. 1603-1610.

VIDAL C., 2005, « Cerveau sexe et idéologie », Diogène, n² 208, PUF, Paris. 